\title{
Progress in Field Spectroscopy
}

\author{
Edward J. Milton ${ }^{1,}$, Nigel P. Fox ${ }^{2}$, Michael E. Schaepman ${ }^{3}$ Member IEEE et al. \\ ${ }^{1}$ School of Geography, University of Southampton, UK, e.j.milton@soton.ac.uk, ${ }^{2}$ National Physical Laboratory, UK, ${ }^{3}$ Centre for \\ Geo-Information, Wageningen University, Netherlands.
}

\begin{abstract}
The measurement of spectral data in the field has an important role in remote sensing, and a long history, but instruments and methods to achieve this have serious limitations under all but the most ideal conditions. Problems arise from the instruments themselves, from the environment in which they are used, and from the methodologies that are commonly adopted.
\end{abstract}

The variable most commonly sought from field measurements is spectral reflectance, or more strictly the bidirectional reflectance factor (BRF), but this is dependent to some extent on the instrument used to make the measurement, and the conditions of measurement, notably the sky irradiance distribution. In this paper we argue that field spectral measurements should be recorded in the appropriate SI units, which will normally be the derived units of radiance for the flux reflected from the target and irradiance for the incident energy. Reflectance data remain a convenient way to represent the energy interactions occurring at the surface, and they have value in generic spectral libraries, but ultimately they lack reproducibility unless accompanied by much more detailed metadata than is the norm in most spectral libraries.

\section{Keywords: field spectroscopy, methodology}

\section{INTRODUCTION}

The earliest uses of field spectroscopy were in energy balance studies [1], investigations into the spectral properties of plants [2] and the quantification of the colour of natural targets observed from aerial platforms [3]. The desire to make measurements independent of the instrument used and of the conditions of measurement quickly led to the adoption of spectral reflectance as the measurand of choice, and several methods were developed to compensate for irradiance variations during measurements. The basis for all quantitative field spectroscopy is the bidirectional reflectance distribution function which was defined by Nicodemus and colleagues $[4,5]$, and which introduced the nomenclature for the subject which is still used today.

\section{THE ROLE OF FIELD SPECTROSCOPY WITHIN EARTH OBSERVATION}

The first role is 'spectroscopy in the field' - the means to make conventional spectroscopic measurements outdoors. The earliest measurements were of leaf reflectance and transmittance, and absorption features in minerals and weathering crusts. Progress in this area has been made possible by advances in electro-optical instrument design and by limiting measurements to small samples under controlled illumination, e.g. the Integrated Spectronics Pima spectroradiometer [6] and the Analytical Spectral Devices contact probe attachment (aka the potato masher) [7]. The state-of-the-art in this area of application is represented by in situ measurements of passive fluorescence and the dynamics of photosynthesis.

The second role is measuring the spectral reflectance of objects in the natural environment. This differs from the first role in that the areas measured are larger (e.g. canopies not leaves) and the illumination is uncontrolled and is a mixture of light directly from the Sun and from the sky. It has taken many years for this role to become fully integrated within the methodology of Earth observation, but this is now an essential part of:

- post-launch vicarious calibration of satellite sensors using extended uniform ground targets $[8,9]$

- practical atmospheric correction (e.g. the Empirical Line method [10])

- validation of atmospheric correction models [11].

- $\quad$ validation of biophysical models (e.g. soil brdf [12], snow brdf [13] and vegetation canopy models [14]).

Progress in this area has been represented by two trends. First, the desire to drive down the uncertainty in individual measurements of spectral reflectance, for example by using dual-beam instruments to measure spectral radiance and irradiance simultaneously [15], and second, by the desire to sample the entire hemisphere of reflected flux, and hence measure the directional reflectance from the surface $[16,17]$. The challenge now is to combine these two approaches into a methodology which will enable high-precision measurements of brdf in the field environment.

The third role is Education and Training. The early spectroradiometers were expensive and complex, but their potential in education was soon recognised, leading to the development of 'hand-held radiometry' [18, 19] based on multiband instruments. There are now relatively low-cost instruments available both for large area measurements [20] and for contact measurements [21].

\section{PROGRESS TOWARDS INCREASING THE REPEATABILITY OF FIELD SPECTROSCOPY}

'Repeatability' in this context relates to the precision of the spectroradiometer and related equipment, and is characterised by the signal-to-noise ratio of spectral measurements. Advances in this area have been driven by improvements in 
electronics, especially detector technology, digital storage, and better optical design. Thermoelectric cooling and techniques to reduce the effect of dark current drift have made it possible to make precise measurements in the field, even in the short-wave infra-red.

\section{PROGRESS TOWARDS IMPROVING THE REPRODUCIBILITY OF FIELD SPECTROSCOPY}

Repeatability is only one aspect of data quality. We must also consider the 'reproducibility' of measurements, which is dependent upon both the inherent signal-to-noise ratio of the spectroradiometer and the methodology used to acquire the data under field conditions. Reflectance data may be acquired using either single-beam or dual-beam methods, and either of these may use a reference panel or a cosine-corrected receptor. There are many sources of uncertainty introduced by the field environment, and these have the potential to significantly degrade the quality of field spectral measurements.

\section{PROGRESS TOWARDS INCREASING THE ACCURACY OF FIELD SPECTROSCOPY}

Accuracy depends upon a clear definition of what is being measured, on the conditions under which it is being measured, and on an unbroken traceability chain with known uncertainty at each step. Many of the sources of uncertainty affecting the repeatability of spectral measurements are understood and may be quantified, but many of those affecting the reproducibility of the measurements remain subject to speculation based upon incomplete knowledge of the radiation environment in which measurements are made.

Although 'spectral reflectance' has a long and distinguished history as the prime measurand of interest to field spectroscopy, the fundamental physical SI units are spectral radiance and spectral irradiance. Although spectral reflectance is often promoted on the basis that it is an inherent property of the surface, this is only partly true. The inherent property of the surface is actually its brdf, and this is impossible to measure under field conditions. As a consequence of this, the accuracy of field spectroscopy is more clearly defined in terms of the traceability of radiance and irradiance measurements in the field environment. The combination of these measurements to create 'reflectance' is a derived measurement, the accuracy of which is to a large part dependent upon the instruments used to make the measurements, the methodology and the conditions of measurement.

\section{A NEW PARADIGM FOR FIELD SPECTROSCOPY?}

The previous discussion has highlighted the problem of treating spectral reflectance as a fundamental physical unit in field spectroscopy. The impossibility of measuring the brdf of surfaces in the field environment has led to many ingenious methods to approximate this parameter, but none of them are entirely satisfactory. An alternative approach would be to focus on radiance and irradiance as the primary physical variables, to develop a traceability chain based around these units, and to regard reflectance as a contingent, derived unit.
This would have a number of advantages. Firstly, the ground data would be directly comparable with the data measured by satellite sensors and therefore more amenable to up-scaling. Secondly, vicarious calibration experiments often conclude that the uncertainty of a radiance-based calibration is less than that of a reflectance-based calibration, largely due to the anisotropy of even the most uniform ground calibration target.

As well as decoupling the target radiance from the irradiance environment it is important to measure the irradiance hemisphere with the same attention to detail given to the target radiance hemisphere. This could be achieved using a sun photometer triggered at the same instant as a goniometer-based spectroradiometer.

On the other hand, more effort will be required to calibrate spectroradiometers to radiance units, and to maintain that calibration over the temperature ranges encountered in the field. The development of dedicated portable calibration devices such as the NPL TSARS system [22] will help address this problem.

On balance, effort devoted to improved methods to measure radiance and irradiance in the field environment would be expected to drive down the uncertainty in derived measurements of reflectance, and thereby improve the accuracy of field spectroscopy

\section{CONCLUSIONS}

The emphasis on spectral reflectance has held back the contribution of field spectroscopy to quantitative EO. However, this has enabled it to perform an invaluable education and training role. Reflectance spectra are so fundamental to how we think that it would be folly to abandon them, but they should come with a 'health warning'. Reflectance data remain a convenient way to represent the energy interactions occurring at the surface, and they have value in generic spectral libraries, but ultimately they lack reproducibility unless accompanied by much more detailed metadata than is the norm in most spectral libraries.

Within the area of quantitative Earth observation, effort now should be devoted to a new paradigm for field spectroscopy in which quantitative, simultaneous measurements of directional radiance and irradiance are the focus of interest.

\section{REFERENCES}

[1] Krinov, E. L. (1953). Spectral reflectance of natural formations, Translation by National Research Council of Canada, Ottawa.

[2] Gates, D. M., H. J. Keegan, et al. (1965). "Spectral properties of plants." Applied Optics 4: 11-20.

[3] Penndorf, R. (1956). Luminous and spectral reflectance as well as colors of natural objects, U.S. Air Force Cambridge Research Center, Bedford, Massachusetts.

[4] Nicodemus, F. E. (1970). "Reflectance nomenclature and directional reflectance emissivity." Applied Optics 9(6): 1474-5.

[5] Nicodemus, F. F., J. C. Richmond, et al. (1977). Geometrical considerations and nomenclature for reflectance, U.S. Govt. Printing Office, Washington D.C. 20402.

[6] http://www.intspec.com/ 
[7] http://www.asdi.com/products-accessories-HICP.asp

[8] Slater, P. N., S. F. Biggar, et al. (1987). "Reflectance- and radiancebased methods for the in-flight absolute calibration of multispectral scanners." Remote Sensing of Environment 22: 11-37.

[9] Thome, K. J. (2001). "Absolute radiometric calibration of Landsat 7 ETM+ using the reflectance-based method." Remote Sensing of Environment 78: 27-38.

[10] Moran, M. S., R. Bryant, et al. (2001). "A refined empirical line approach for reflectance factor retrieval from Landsat-5 TM and Landsat-7 ETM+." Remote Sensing of Environment 78: 71-82.

[11] Guzzi, R. and G. C. Maracci, Rizzi,R., and Siccardi,A. (1985). "Spectroradiometer for ground-based atmospheric measurements related to remote sensing in the visible from a satellite." Applied Optics 24: 2859-2864.

[12] Cierniewski, J., T. Gdala, et al. (2004). "A hemispherical-directional reflectance model as a tool for understanding image distinctions between cultivated and uncultivated bare surfaces." Remote Sensing of Environment 90: 505-523.

[13] Painter, T. H., B. Paden, et al. (2003). "Automated spectro-goniometer : A spherical robot for the field measurement of the directional reflectance of snow." Review of Scientific Instruments 74(12): 5179-5188.

[14] Goel, N. S. and N. E. Reynolds (1989). "Bidirectional canopy reflectance and its relationship to vegetation characteristics." International Journal of Remote Sensing 10: 107-132.

[15] Anderson, K. and E. J. Milton (2006). "On the temporal stability of ground calibration targets: implications for the reproducibility of remote sensing methodologies." International Journal of Remote Sensing.

[16] Deering, D. W. and P. Leone (1986). "A sphere-scanning radiometer for rapid directional measurements of sky and ground radiance." Remote Sensing of Environment 19: 1-24.

[17] Sandmeier, S. and K. Itten (1999). "A Field Goniometer System (FIGOS) for Acquisition of Hyperspectral BRDF Data." IEEE Transactions on Geoscience and Remote Sensing 37(2): 978-986.

[18] Milton, E. J. (1980). "A portable multiband radiometer for ground data collection in remote sensing." International Journal of Remote Sensing 1: 153-165.

[19] Tucker, C. J. (1978). Hand-held radiometer studies of vegetation in-situ: a new and promising approach. Proceedings of an International Symposium on Remote Sensing for Observation and Inventory of Earth Resources and the Endangered Environment, Freiburg, West Germany, 2-8 July, ISP Commission VII and IVFRO Subject Group 6.05; International Archives of Photogrammetry.

[20] http://www.oceanoptics.com/

[21] http://www.lpi.usra.edu/education/products/spectrometer/

[22] Woolliams, E. R., Hunt, T. M., Harrison, N. J., Windsor, S. A., Fox, N. P., R. Mountford, J., Rogers, L. J. and Pegrum, H. M. (2002). Improved Transfer Standard Sources for Calibration of Field Spectrometers Used for Earth Observation Applications, 9th International Symposium on Remote Sensing, Sensors, Systems, and Next-Generation Satellites VI, SPIE. 4881, Aghia Pelagia, Crete, Greece. 386-394 